\title{
Metodologias Ativas como forma de reduzir os desafios do ensino em Engenharia de Software: diagnóstico de um survey
}

\author{
José Vinícius V. Lima ${ }^{1}$, Cleverton Silva ${ }^{1}$, Fernanda Alencar ${ }^{1,2}$, Wylliams Santos ${ }^{1}$ \\ ${ }^{1}$ Universidade de Pernambuco - (UPE) \\ ${ }^{2}$ Universidade Federal de Pernambuco - (UFPE) \\ \{jvvl,cads\}@ecomp.poli.br, fernanda.ralencar@ufpe.br, wbs@upe.br
}

\begin{abstract}
One of the challenges of teaching Software Engineering (SE) in Higher Education Institutions (HEI) is to adapt teaching models that can meet the demands of a dynamic market and assign skills to students through practical situations. Active Methodologies (AMs) have been used in teaching SE as a way to promote innovative, humanistic, critical, and reflective training. Thus, this article diagnosed how AMs are being applied in the teaching of SE in HEI in Brazil. For this, a survey was applied with 60 teachers from the 5 regions of the country. Factors and skills needed for the use of AMs were identified. Besides, a set of lessons learned and challenges faced was categorized.
\end{abstract}

Resumo. Um dos desafios do ensino da Engenharia de Software (ES) nas Instituições de Ensino Superior (IES) é adaptar modelos de ensino que possam atender às demandas de um mercado dinâmico e atribuam competências aos discentes através de situações práticas. Metodologias Ativas (MAs) têm sido utilizadas no processo do ensino da ES como forma de promover uma formação inovadora, humanística, crítica e reflexiva. Assim, este artigo diagnosticou como as MAs estão sendo aplicadas no ensino da ES em IES do Brasil. Para isso, um survey foi aplicado com 60 docentes das 5 regiões do país. Fatores e habilidades necessárias para o uso de MAs foram identificados. Além disso, categorizou-se um conjunto com lições aprendidas e desafios enfrentados.

\section{Introdução}

A Engenharia de Software (ES) consiste na aplicação sistemática de conhecimento científico e tecnológico, métodos e experiências ao projeto, implementação, teste e documentação [Pressman and Maxim 2016]. A ES se apresenta como uma das disciplinas de maior relevância na área da computação [ACM/IEEE 2014]. Segundo Cunha et al. (2018), essa disciplina detém denso conteúdo teórico-conceitual, baseando-se no ensino tradicional com aulas expositivas. Paradigmas e tendências surgem para atender às exigências da sociedade e de um mercado dinâmico. Assim, a educação tenta adequar-se às decorrências desse progresso. Por isso o processo de ensino da ES, nas Instituições de Ensino Superior (IES) é tido como um desafio [Ferreira et al. 2018].

Além do aspecto teórico, os alunos necessitam adquirir habilidades técnicas a serem praticadas em suas carreiras [Souza et al. 2019]. Isso é o que enfatiza estudos recentes para o desenvolvimento das competências esperadas nos profissionais da ES. Conforme Lima et al. (2019), aptidões como liderança, negociação, tomada de decisão 
IX Congresso Brasileiro de Informática na Educação (CBIE 2020)

Anais do XXXI Simpósio Brasileiro de Informática na Educação (SBIE 2020)

e auto-reflexão são tidas como fundamentais no processo de ensino-aprendizagem da ES, uma vez que, o desenvolvimento de software também envolve aspectos humanos e sociais.

Ao buscar alinhar a teoria com a prática, o processo de ensino-aprendizagem da ES deve incluir habilidades e atitudes que vão além de conceitos, técnicas e métodos. Assim, docentes e pesquisadores têm buscado criar ambientes motivacionais em suas disciplinas para superarem as fronteiras do ensino tradicional. Neste sentido, Lima et al. (2019) manifestam que o uso de abordagens centradas no aluno podem ser mais adequadas para permitir o desenvolvimento de competências baseadas na ação-reflexão-ação. Proporcionando desta forma, elevação da autonomia e estímulo ao pensamento crítico.

Deste modo, é fundamental que outras metodologias de ensino-aprendizagem sejam utilizadas em paralelo às aulas expositivas para que os alunos vivenciem experiências similares àquelas de sua futura atuação profissional [Souza et al. 2019]. Com isso, a educação no contexto contemporâneo perpassa, entre outros aspectos, pela discussão sobre as Metodologias Ativas (MAs) produzidas de forma colaborativa e as suas implicações nas experiências vivenciadas pelos discentes em sala de aula [Bacich and Moran 2018].

Diante desse cenário, as MAs têm surgido como alternativas de ensino em várias áreas. Tem-se introduzido as MAs no processo de ensino-aprendizagem em disciplinas da ES como oportunidade de inovar, propor novas formas de ensinar, estimular competências voltadas a experiências reais e aproximar a academia da indústria [Ferreira et al. 2018]. Apesar da relevância de tais propostas para a melhoria do processo de ensino-aprendizagem na ES, é importante analisar o contexto no qual as metodologias ativas estão sendo utilizadas pelos docentes na área do ensino da ES.

Para tratar desse aspecto, este trabalho tem por objetivo diagnosticar o uso de MAs no processo de ensino-aprendizagem das disciplinas da ES nas IES brasileiras. Para isso, foi realizado um survey com docentes especialistas no uso de MAs em disciplinas da ES no Brasil. Dados quantitativos e qualitativos foram coletados e análise qualitativa foi aplicada. Observou-se fatores que levam os docentes a utilizar as MAs e o grau de relevância ao introduzi-las no ensino em ES. Identificou-se também 14 categorias relacionadas às lições aprendidas ao fazer o uso de MAs e um conjunto com 13 desafios enfrentados.

Além dessa Seção introdutória, este trabalho apresenta a Seção 2 que discorre sobre a contextualização desta pesquisa. Na Seção 3, toda a estrutura do survey é retratada. A Seção 4 relata os dados obtidos ao conduzir o survey. Os resultados são discutidos e as limitações são expostas na Seção 5 . Por fim, a Seção6 6 apresenta as considerações finais.

\section{Ensino de Engenharia de Software e Metodologias Ativas}

O ensino da ES deve incluir experiências aos discentes com a prática profissional, permitindo que os futuros profissionais cheguem ao mercado de trabalho preparados para enfrentar os desafios da dependência crítica da sociedade em relação ao software [Ardis et al. 2015]. Diante do exposto, Ferreira et al. (2018) salientam que a ES precisa ser ensinada utilizando outras alternativas além dos métodos tradicionais.

Com isso, Souza e França (2020) reforçam sobre a mudança de paradigma a qual vem sendo adotada no processo de ensino-aprendizagem da ES. Ou seja, a transformação do formato de aulas expositivas para modelos de ensino com metodologias que promovam uma formação humanística, crítica e reflexiva às novas gerações de estudantes e futuros 
IX Congresso Brasileiro de Informática na Educação (CBIE 2020)

Anais do XXXI Simpósio Brasileiro de Informática na Educação (SBIE 2020)

profissionais da ES. Neste aspecto, pesquisadores da área evidenciam as MAs como alternativas mais indicadas no processo de ensino-aprendizagem em ES.

Segundo Bacich e Moran (2018), MAs são processos de interação de conhecimentos, análises e decisões, nos quais o docente age como mediador e o discente como gestor do conhecimento. Para Diesel et al. (2017), as MAs exigem dos alunos ações e construções variadas, estímulos ao pensamento crítico, resoluções de problemas reais e auxílio na tomada de decisões, o que costumam acarretar em habilidades e melhorias para o desenvolvimento intelectual, educacional e profissional aos discentes da ES.

As MAs são fundamentadas por um conjunto de princípios relacionados com a aprendizagem construtiva, colaborativa, interdisciplinar, reflexiva, crítica, investigativa, humanista, motivadora e desafiadora [Mourão 2017]. A partir desse conceito, são exemplos de MAs: Aprendizagem Baseada em Problemas (PBL), estudos de casos, Aprendizagem Baseada em Projetos (PjBL), sala de aula invertida, mapas mentais e conceituais, Aprendizagem Baseada em Equipes, discussões em classe, gamificação, entre outras.

\section{O Survey}

Survey é um método de pesquisa abrangente que é executado com o objetivo de obter, descrever ou explicar conhecimentos das pessoas, buscando entender determinado aspecto de uma população [Wohlin et al. 2012]. O objetivo do estudo apresentado neste trabalho foi compreender como as MAs estão sendo colocadas em prática pelos docentes das disciplinas da ES em IES brasileiras. Para isso, uma abordagem descritiva foi utilizada para coletar e explorar aspectos sobre as opiniões dos docentes, representados por uma amostra, com relação ao uso de MAs no processo de ensino-aprendizagem da ES.

Para atingir o objetivo, este survey tem como finalidade descobrir: (i) qual a percepção dos docentes sobre o uso de MAs no processo de ensino-aprendizagem da ES; (ii) quais MAs estão sendo utilizadas no ensino da ES; (iii) quais fatores, competências, habilidades e conhecimentos são importantes para utilizar as MAs; (iv) quais são os aspectos positivos e lições aprendidas identificadas pelos docentes ao usarem as MAs e (v) quais são os problemas e desafios observados pelos docentes quanto ao uso de MAs.

\subsection{Planejamento}

Para responder às questões supracitadas, um instrumento de pesquisa foi desenvolvido e seguiu os guidelines propostos por Kitchenham e Pfleeger (2008). Neste processo, um questionário auto administrado e classificado como cross-sectional (transversal) foi criado contento 17 questões nas quais foram divididas em dois grupos: questões discursivas e objetivas. O primeiro grupo permitiu que os participantes descrevessem suas respostas de forma completa através de um espaço disponível para livre resposta textual sem qualquer padrão exigido. Já o segundo utilizou questões para uniformizar as respostas, abrangendo alternativas de múltipla escolha e perguntas baseadas em Likert Scales com a seguinte escala ordinal definida: quase nunca útil, ocasionalmente útil, moderadamente útil, muito útil e essencial. Essa escala equivale, respectivamente, de 1 a 5 graus de concordância.

Os dados relacionados às questões discursivas foram analisados através de técnicas da Grounded Theory (GT). A GT propõe-se criar uma teoria a partir dos dados coletados e analisados sistematicamente, a mesma é composta por três fases: (i) codificação 
aberta; (ii) codificação axial e (iii) codificação seletiva [Corbin and Strauss 2014]. Visando a identificação de tópicos associados às lições aprendidas e aos desafios enfrentados, esta pesquisa utilizou-se as fases I e II da GT para analisar os dados. A ferramenta de análise qualitativa Atlas.ti $\left.\right|^{1}$ foi utilizada como forma de apoiar esse procedimento. A estatística descritiva foi utilizada para representar e descrever os dados de caracterização dos participantes com relação às questões objetivas.

\subsection{Público-alvo}

Como este estudo está relacionado à procura de evidências associadas ao uso de MAs no processo de ensino-aprendizagem da ES em IES, considerou-se como público-alvo do survey docentes que atuam no ensino da ES em cursos de nível superior no Brasil. A fim de garantir que estes participantes fornecessem informações confiáveis aos objetivos traçados nesta pesquisa, foram incluídos docentes que atuam em IES pública ou privada brasileira a qual segue as diretrizes curriculares da área da Ciência da Computação. Foram excluídos os participantes que não se apresentavam motivados a participar da pesquisa ou, ainda, aqueles que não estavam relacionados com área do ensino da ES.

\subsection{Instrumento de Pesquisa}

Como instrumento de pesquisa e aplicação do survey, o questionário criado para coleta dos dados foi estruturado em três seções: (i) apresentação do contexto da pesquisa e o Termo de Consentimento Livre e Esclarecido (TCLE); (ii) informações sobre o perfil profissional do participante e (iii) uso de MAs no processo de ensino-aprendizagem da ES. A primeira seção é composta pela descrição do contexto da pesquisa, mencionando a justificativa, os objetivos e a apresentação do TCLE ao responsável. A segunda parte buscou incluir questões demográficas com a finalidade de caracterizar os participantes. Já na terceira seção, as questões procuravam analisar a visão dos docentes com relação ao uso de MAs no processo de ensino-aprendizagem da ES.

Para definir as perguntas deste questionário, pesquisas na literatura sobre o contexto do ensino-aprendizagem em ES foram realizadas a fim de identificar estudos relevantes perante a área coberta nesta pesquisa. Algumas das questões foram baseadas no estudo realizado por Kitchenham et al. (2005). O questionário (que pode ser acessado através deste $\operatorname{lin} \sqrt{2}^{2}$ é do tipo eletrônico e foi disponibilizado na web de forma on-line utilizando a ferramenta Google Forms. Antes da coleta dos dados, o mesmo foi avaliado por três professores-pesquisadores na área da ES e especialistas no uso de MAs. Após isso, ajustes foram realizados e o tempo médio gasto para responder o survey foi registrado.

\subsection{Execução}

A realização do survey ocorreu no período de 8 a 25 de junho de 2020. O questionário foi submetido diante do público-alvo já mencionado. A fim de reduzir o viés da amostragem, o survey foi aplicado em todas regiões do país. O questionário foi enviado via e-mail para 302 participantes e 60 responderam, obtendo-se um percentual de respostas de 19,9\%. Conforme Nulty (2008), em estudos do tipo surveys on-line, essa taxa de respostas é considerada como satisfatória. Deste modo, foram registradas respostas de 30 IES brasileiras, sendo 25 públicas e 5 privadas. Essas IES representam 13 estados do Brasil, sendo 45\% da região Sul, 35\% do Nordeste, $10 \%$ do Centro-Oeste, $8,3 \%$ do Sudeste e 1,7\% do Norte.

\footnotetext{
${ }^{1}$ https://atlasti.com/

${ }^{2} \mathrm{https} / / /$ bit.ly/InstrumentodePesquisa
} 


\section{Resultados da Aplicação do Survey}

Nesta Seção, apresenta-se os dados obtidos após a execução do survey. A ideia é a construção de um panorama sobre as práticas do ensino da ES em IES brasileiras.

\subsection{Perfil dos Docentes}

Os docentes que responderam à pesquisa possuem formação acadêmica em 11 áreas distintas. Com destaque para formação em Ciência da Computação a qual registrou $65 \%$ das respostas, seguido da Engenharia de Software com $10 \%$ e Processamento de Dados com 8,3\%. Além disso, cerca de 53,4\% dos participantes são doutores e $61,7 \%$ dos mesmos possuem experiência acima de 10 anos na docência. As demais informações sobre os diferentes níveis de formação juntamente com o tempo de experiência na docência podem ser identificadas ao observar a Figura 1 .

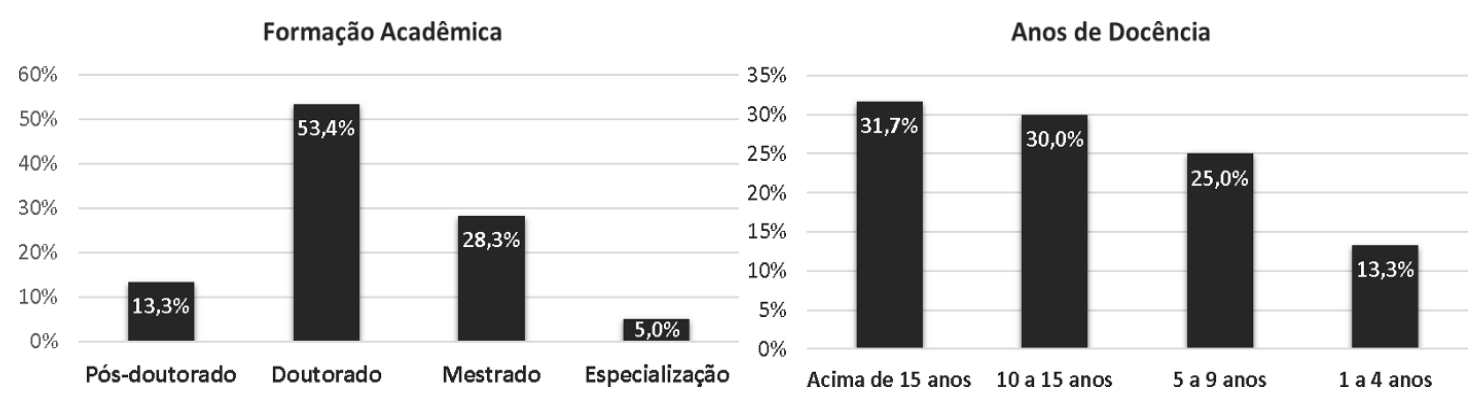

Figura 1. Formação acadêmica e tempo de experiência entre os participantes.

Outro aspecto identificado foi o tipo de abordagem de ensino que os docentes costumavam utilizar no processo de ensino das disciplinas da ES. Desta forma, observouse que 78,4\% fazem o uso da abordagem híbrida, a qual adapta o ensino da ES com interações que combinam a teoria com a prática, acrescentando o uso de MAs a fim de gerar competências voltadas às atuações no mercado de trabalho, bem como a aplicabilidade da ES em situações da vida real. Cerca de 13,3\% são adeptos apenas à abordagem tradicional com aulas teóricas e expositivas e 8,3\% utilizam a abordagem ativa na íntegra.

Paralelamente, foi possível descobrir a relação entre a formação acadêmica dos participantes com o uso de MAs. Assim, os seis tipos de formações mais apontadas foram analisadas e mapeadas de acordo com as MAs que cada grupo costuma utilizar no ensino da ES. A Figura 2 expõe a relação de causa e efeito entre essas duas perspectivas.

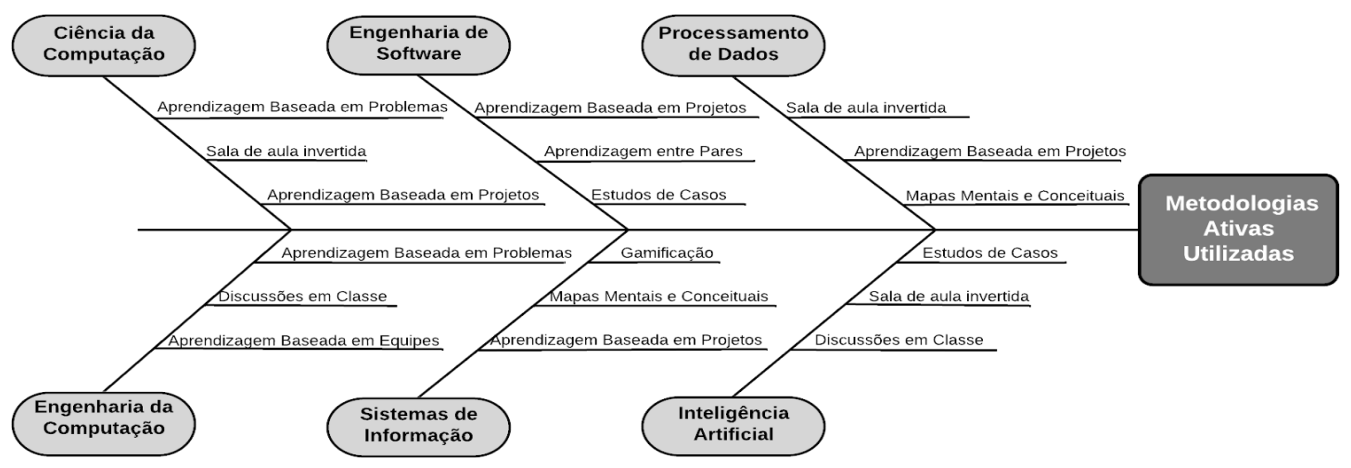

Figura 2. Relação de causa e efeito entre a formação acadêmica e o uso de MAs. 


\subsection{Metodologia Ativas e sua Relevância no Ensino de Engenharia de Software}

Com base nos estudos que enfatizam a necessidade em propiciar aos alunos da ES experiências práticas para ascendência de competências direcionadas às habilidades requeridas na indústria de software, procurou-se analisar as opiniões dos participantes com relação à utilidade em aprender mais sobre o uso de MAs no ensino da ES, buscando por cursos adicionais, por exemplo. Com isso, constatou-se que os docentes têm buscado aprimorar seus métodos de ensino, uma vez que, 76,7\% dos mesmos afirmaram que é muito útil ou essencial a procura por novos conhecimentos quanto ao uso de MAs. Da mesma forma, foi avaliado a relevância em utilizar MAs no ensino da ES. Os resultados sinalizaram que $85 \%$ dos participantes declararam como primordial a utilização de MAs nas disciplinas da ES. A Figura 3 traz a análise das informações em detalhes.

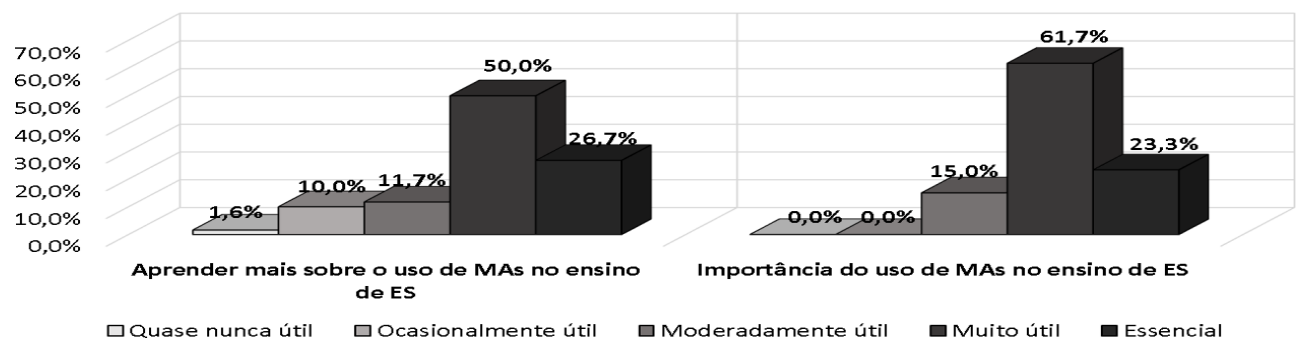

Figura 3. Utilidade no aprendizado de MAs e sua importância no ensino em ES.

Esses dados salientam a importância em modificar o modelo de ensino em ES com estratégias que possam atenuar as dificuldades existentes atualmente. Como também o interesse significativo que os docentes dispõem na busca do aprendizado de MAs. Segundo um docente: "aprender sobre MAs é essencial, estas exigem inovação na preparação dos materiais, planejando com cuidado os componentes que serão trabalhados a fim de ter metas muito bem definidas do progresso e do desenvolvimento de habilidades".

\subsection{Tipos de Metodologias Ativas e Fatores que Provocam o Uso}

Quanto aos tipos de MAs utilizadas pelos docentes, observou-se que a PjBL $(68,3 \%)$ tem sido amplamente aplicada no processo de ensino-aprendizagem em ES, seguida da PBL $(61,7 \%)$ e discussões em classe (55\%). Essas MAs refletem em propostas que são baseadas em situações do mundo real, aproximando a academia da indústria de software, o que impacta no desenvolvimento de competências aos discentes ligadas às habilidades provenientes do cotidiano profissional. A Figura 4 apresenta as demais MAs identificadas.
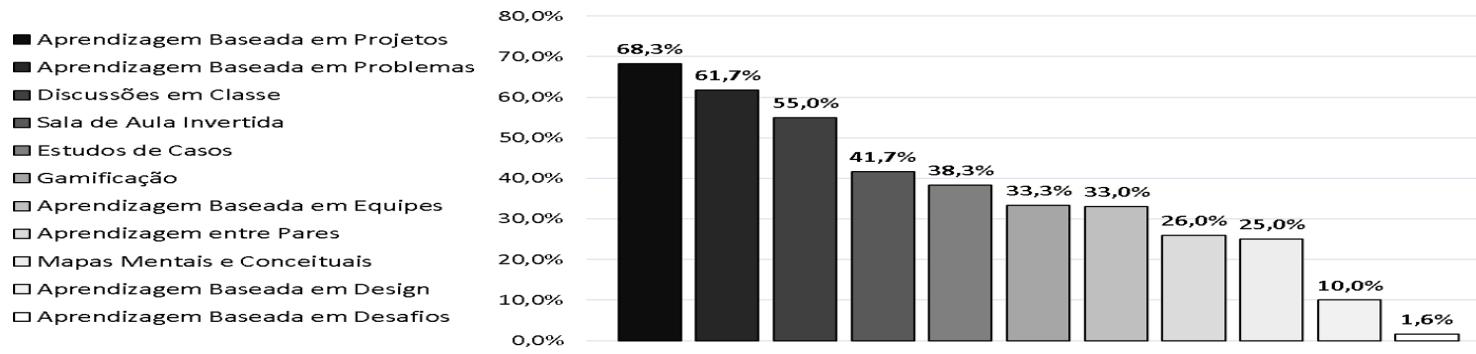

Figura 4. Metodologias ativas identificadas.

Após identificar as MAs utilizadas, buscou-se pelos motivos que os docentes estabelecem para inseri-las no ensino da ES. Os participantes deveriam selecionar uma ou 
mais entre as 11 opções preestabelecidas, assim como inserir um outro fator, caso entendessem como necessário. Ao final da execução do survey, foram mencionadas outras 6 condições, totalizando assim em 17 motivações. A Figura 5 apresenta os cinco fatores mais apontados pelos participantes. Entre as circunstâncias apontadas pelos mesmos, destaca-se o seguinte comentário: "o uso de MAs torna o conteúdo tão atrativo aos discentes, criando um ambiente inovador, que a IES modificou a grade curricular do curso, introduzindo o uso de MAs como parte obrigatória na emenda do Projeto do Curso".

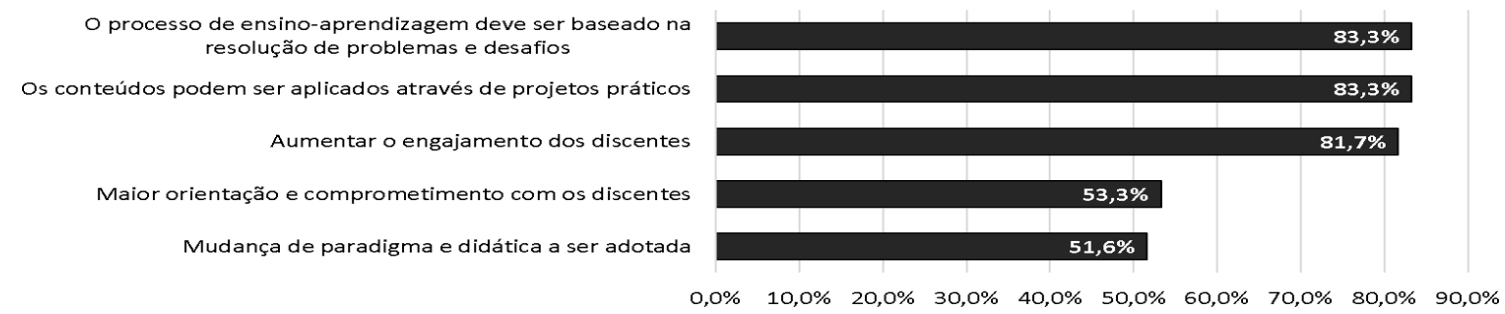

Figura 5. Principais fatores que ocasionam o uso de MAs no ensino em ES.

\subsection{Lições Aprendidas e Desafios Identificados ao utilizar as Metodologias Ativas}

Conforme mencionado na Subseção 3.1, técnicas da Grounded Theory foram aplicadas buscando realizar a análise qualitativa com base nas respostas obtidas das questões discursivas. Na codificação aberta, os dados foram analisados detalhadamente buscando criar códigos relacionados a trechos específicos de cada resposta. Já a codificação axial buscou identificar as categorias e criar relacionamentos entre os códigos. Com o objetivo de evitar viés a pesquisa, todo esse processo foi realizado e discutido em dupla.

Em relação às lições aprendidas, constatou-se um conjunto com 14 categorias. A Figura 6 apresenta a rede formada entre elas. Por meio da análise, identificou-se que, ao adotar a abordagem híbrida, a mesma está concatenada a um acompanhamento contínuo das atividades realizadas, além da criatividade. Com isso, o docente necessita aperfeiçoar o seu perfil de atuação, sendo essencial fazer o uso de materiais extraclasse, como também possuir experiência no assunto a ser abordado. Adicionalmente, verificou-se que, ao introduzir o uso de MAs no ensino da ES, as mesmas desenvolvem a aquisição de novas habilidades e mudanças de comportamento nos discentes. Essas transmutações impactam no maior comprometimento e engajamento, aumentando o interesse, motivação e participação, promovendo assim, a elevação da aprendizagem.

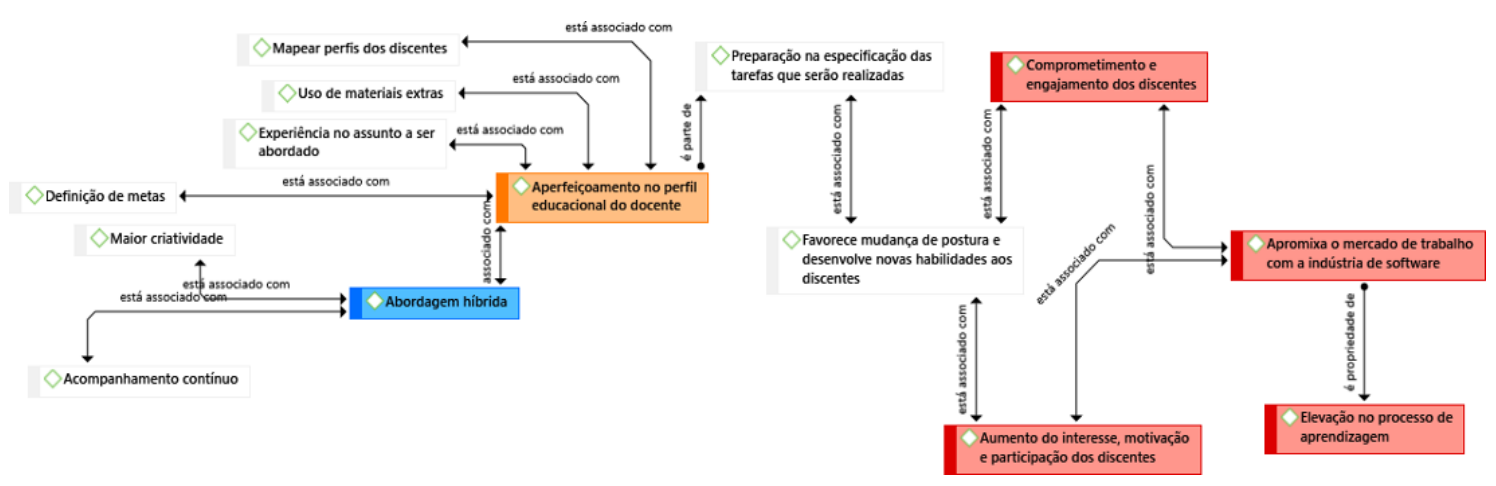

Figura 6. Categorias relacionadas às lições aprendidas ao utilizar as MAs. 
Também foram identificadas 13 categorias relacionadas aos desafios enfrentados pelos docentes ao usar as MAs. A Figura 7 apresenta o detalhamento das mesmas junto com seus relacionamentos. Pode-se observar dificuldades associadas à mudança de paradigma a qual deve ser adotada, o que acarreta em uma carência na preparação pedagógica do docente. Devido a isso, faz-se necessário uma modificação e preparação no ambiente educacional da ES, provocando uma transformação na postura dos discentes, dado que estes tendem a resistir a possíveis mudanças que possam fugir do ensino tradicional. Assim, há um impacto na adaptação dos mesmos quando as MAs são incluídas no processo de ensino-aprendizagem em ES. Além do excesso de tempo que as mesmas provocam, em razão de, muitas vezes, os conteúdos serem ministrados em apenas um semestre.

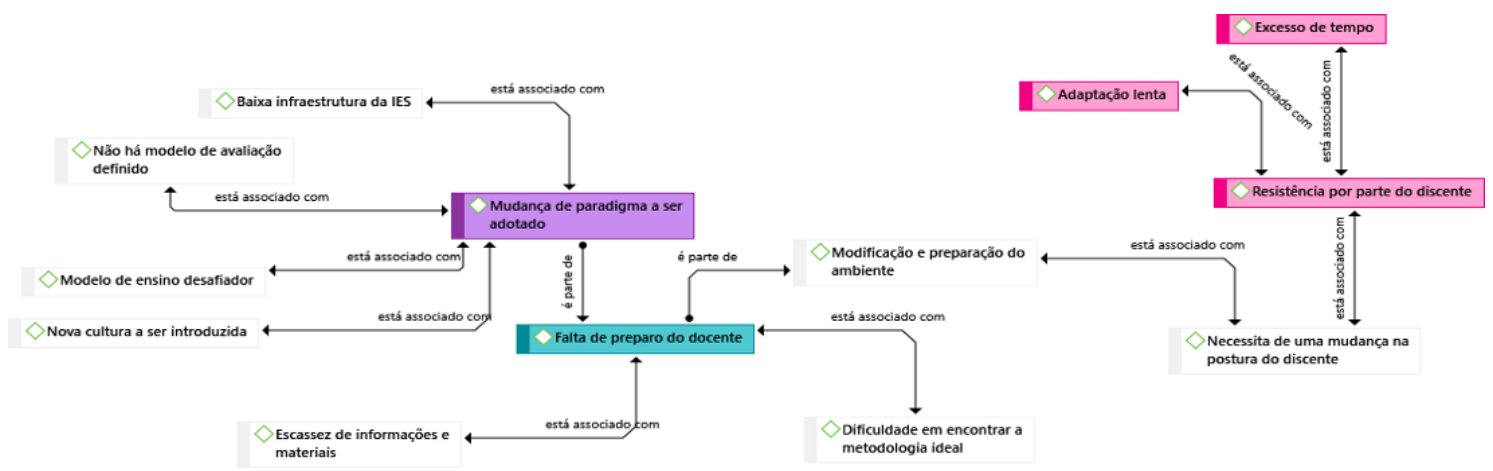

Figura 7. Categorias relacionadas aos desafios identificados no uso de MAs.

\section{Discussão do Diagnóstico}

Os resultados obtidos possibilitaram realizar uma análise no contexto do ensino da ES, mais especificamente, investigando como as MAs estão sendo inseridas e de qual forma as mesmas são utilizadas pelos docentes da ES em IES brasileiras. Deste modo, o diagnóstico do survey expõe as necessidades relatadas pelos docentes referente à criação de novas estratégias voltadas ao ensino em ES. Além disso, $85 \%$ dos docentes apontaram a utilização de MAs como mudanças positivas para as práticas educacionais na área da ES.

Tais números refletem na predominância referente à adoção da abordagem híbrida no ensino da ES atualmente. Esse modelo costuma mesclar aspectos teóricos e práticos, compondo-se por meio do uso de MAs e impulsionando o conhecimento através de atividades provenientes de situações do mundo real. De acordo com um dos participantes: " $o$ modelo híbrido agiliza o aprendizado e prepara o discente para o que ele vai encontrar no mercado de trabalho". Essa afirmação vai ao encontro com os principais tipos de MAs utilizadas no ensino da ES, dado que, as duas mais aplicadas são MAs que fazem uso de projetos e problemas reais, provocando em aptidões exigidas pelas indústrias de software.

Com a ascendência voltada ao uso da abordagem híbrida, os resultados elucidam que os docentes da ES têm procurado aperfeiçoar seus perfis pedagógicos, sobretudo, no tocante à busca do aprendizado sobre as MAs. Desta maneira, os docentes relataram que o uso de MAs no ensino da ES exige maior preparação e planejamento prévio dos mesmos, sendo necessário estudar o perfil dos alunos, definir metas, inovar na preparação dos materiais e especificar de forma detalhada os requisitos das tarefas que serão feitas.

Neste sentido, os participantes destacaram fatores que provocam a utilização de MAs no ensino da ES. Os resultados destacaram a ênfase para um processo de ensino 
embasado através de problemas, desafios e projetos práticos, além do desejo em aumentar o engamento dos discentes. Esse práxis motiva a transformação da didática do docente, provocando um novo paradigma que deve ser seguido. Conforme um participante que atua no ensino da ES há mais de 2 décadas e faz uso de MAs há quase 5 anos: "aplicar MAs converte em uma forma de aumentar o engajamento, uma vez que os alunos que hoje chegam à Universidade se dispersam facilmente e precisam de estratégias de ensino diferentes para reter a atenção e manter o interesse pelo aprendizado".

Apesar do aumento na aplicação de MAs no ensino da ES e os benefícios produzidos pelas mesmas, os resultados indicam alguns obstáculos que tornam difícil o seu uso, sendo eles: baixa infraestrutura da IES, escassez de materiais de apoio, falta de modelo de avaliação definido, consumo de tempo demasiadamente, adaptação lenta e resistência por parte dos discentes, nova cultura a ser introduzida na sala de aula, maior planejamento, orientação e acompanhamento das atividades a serem executadas, o que transfiguram-se em uma estratégia de ensino desafiadora, principalmente a depender do tamanho da turma.

\subsection{Limitações e Ameaças à Validade}

Embora a pesquisa tenha sido realizada cuidadosamente, há algumas limitações e ameças à validade que devem ser consideradas: (i) a taxa de respostas pode não representar toda a população-alvo; (ii) não há garantia de que todos os e-mails enviados eram válidos e que foram recebidos; (iii) o questionário foi impossibilitado de ser disponibilizado in loco devido à pandemia causada pelo Covid-19 e (iv) a definição do instrumento de pesquisa pode ter limitado a pesquisa ou não ter abordado todos os conteúdos relevantes do tema.

\section{Considerações Finais}

Os novos paradigmas adotados pelos docentes demonstram que o atual cenário do ensino em ES nas IES brasileiras tem se modificado. Acredita-se que essa mudança decorre mediante um mercado de trabalho cada vez mais dinâmico, onde o desenvolvimento de habilidades e competências profissionais são tidas como fundamentais para a formação dos discentes. Assim, aplicar MAs no ensino da ES torna-se um diferencial para a obtenção dessas atribuições, amplia o processo de aprendizagem e fortalece o comprometimento.

Este artigo apresentou um estudo que investigou como as MAs estão sendo aplicadas no ensino da ES em IES do Brasil. Para isso, um survey foi aplicado com docentes de disciplinas da ES e obteve respostas de 30 IES públicas e privadas de todas as cinco regiões do país. A partir da análise realizada, foi possível constatar o nível de conhecimento, habilidades, fatores, lições aprendidas e desafios ao usar MAs no ensino da ES.

Para trabalhos futuros, pretende-se propor um guia com base na abordagem híbrida para que os docentes da ES possam ter maior orientação na aplicação de MAs diante de competências específicas e unidades de conhecimentos que desejem aplicar em salas de aula. Espera-se, desta forma, facilitar o planejamento e o uso de MAs por parte dos docentes, além de disseminar novas abordagens e estratégias ativas no ensinoaprendizagem. Nesta perspectiva, estima-se que os discentes possam ter maior engajamento e motivação, revigorando sua produtividade e o desenvolvimento de habilidades.

\section{Agradecimentos}

Os autores gostariam de agradecer à Coordenação de Aperfeiçoamento de Pessoal de Nível Superior (CAPES) pelo apoio financeiro ao desenvolvimento desta pesquisa. 
IX Congresso Brasileiro de Informática na Educação (CBIE 2020)

Anais do XXXI Simpósio Brasileiro de Informática na Educação (SBIE 2020)

\section{Referências}

ACM/IEEE (2014). Curriculum guidelines for undergraduate degree programs in software engineering.

Ardis, M., Budgen, D., Hislop, G. W., Offutt, J., Sebern, M., and Visser, W. (2015). Se 2014: Curriculum guidelines for undergraduate degree programs in software engineering. Computer, (11):106-109.

Bacich, L. and Moran, J. (2018). Metodologias ativas para uma educação inovadora: uma abordagem teórico-prática. Penso Editora.

Corbin, J. and Strauss, A. (2014). Basics of qualitative research: Techniques and procedures for developing grounded theory. Sage publications.

Cunha, J. A. O., Marques, G. A., Lemos, W. L., Câmara Jr, U. D., and Vasconcellos, F. J. (2018). Software engineering education in brazil: a mapping study. In Proceedings of the XXXII Brazilian Symposium on Software Engineering, pages 348-356.

Diesel, A., Baldez, A. L. S., and Martins, S. N. (2017). Os princípios das metodologias ativas de ensino: uma abordagem teórica. Revista Thema, 14(1):268-288.

Ferreira, T., Viana, D., Fernandes, J., and Santos, R. (2018). Identifying emerging topics and difficulties in software engineering education in brazil. In Proceedings of the XXXII Brazilian Symposium on Software Engineering, pages 230-239.

Kitchenham, B., Budgen, D., Brereton, P., and Woodall, P. (2005). An investigation of software engineering curricula. Journal of Systems and Software, 74(3):325-335.

Kitchenham, B. A. and Pfleeger, S. L. (2008). Personal opinion surveys. In Guide to advanced empirical software engineering, pages 63-92. Springer.

Lima, J. V., Júnior, M. d. M. A., Moya, A., Almeida, R., Anjos, P., Lencastre, M., Fagundes, R. A. d. A. F., and Alencar, F. (2019). As metodologias ativas e o ensino em engenharia de software: uma revisão sistemática da literatura. In Anais do Workshop de Informática na Escola, volume 25, page 1014.

Mourão, A. (2017). Uma proposta da eficiência do uso da metodologia ativa baseada em problemas, utilizando dojo de programação, aplicada na disciplina de lógica de programação. In Anais do Workshop de Informática na Escola, volume 23, page 667.

Nulty, D. D. (2008). The adequacy of response rates to online and paper surveys: what can be done? Assessment \& evaluation in higher education, 33(3):301-314.

Pressman, R. and Maxim, B. (2016). Engenharia de Software- $8^{a}$ Edição. McGraw Hill Brasil.

Souza, M. and França, C. (2020). O que explica o sucesso de jogos no ensino de engenharia de software? uma teoria de motivação. In Anais do XXIV Workshop sobre Educação em Computação, pages 260-269. SBC.

Souza, M., Moreira, R., and Figueiredo, E. (2019). Students perception on the use of project-based learning in software engineering education. In Proceedings of the XXXIII Brazilian Symposium on Software Engineering, pages 537-546.

Wohlin, C., Runeson, P., Höst, M., Ohlsson, M. C., Regnell, B., and Wesslén, A. (2012). Experimentation in software engineering. Springer Science \& Business Media. 